Sección dos: Textos

Radiografía de la innovación educativa en el Siglo XXI

\title{
El papel de la inspección de educación en los procesos de innovación educativa ${ }^{1}$
}

The role of education inspectorate in innovation processes

\author{
Manuel Zulueta Castañeda \\ Inspección General de \\ Educación \\ Consejería de Educación y \\ Deporte de la Junta de Andalucía \\ mzulueta@telefonica.net
}

\section{Resumen}

El papel de la Inspección Educativa en relación con la innovación educativa ha supuesto históricamente un rol complejo de asumir debido a las funciones que se le asigna y el modo en que éstas se desarrollan. En este artículo intentaremos proporcionar, partiendo de un breve recorrido por el desarrollo histórico y de la realidad actual de la Inspección de Educación en España y Europa, una propuesta de futuro que permita a esta institución detectar, promover, participar y extender las innovaciones que se desarrollan en los centros educativos.

Palabras clave: Asesoramiento, cambio metodológico, educación, evaluación, innovación educativa, inspección educativa, supervisión.

\begin{abstract}
The role of the Educational Inspection in relation to innovation has historically supposed a complex role to assume due to the functions assigned to it and the way in which they are developed. In this article we will try to provide, based on a brief tour of the historical development and the current reality of the Education Inspectorate in Spain and Europe, a
\end{abstract}

${ }^{1}$ Recibido: 27/11/2019 Evaluado: 20/12/2019 Aceptado: 09/02/2020 
proposal for the future that allows this institution to detect, promote, participate and extend the innovations that are developed in educational establishments.

Keywords: Advice, methodological change, education, evaluation, educational innovation, educational inspection, supervision.

\section{Los antecedentes}

Desde el inicio de la historia de la Inspección de Educación el papel que le han asignado las distintas regulaciones normativas han determinado tanto las funciones de esta institución como el modo en que éstas se han desarrollado.

De este modo, es en la Constitución Española de 1812 cuando por primera vez en una norma de rango superior se hace referencia a la Inspección, fijándose como su función principal el control y la inspección de la marcha real de los establecimientos escolares, si bien este papel era ejercido por las autoridades locales con la ayuda de la Dirección General de Estudios.

Será con el Real Decreto de 30 de marzo de 1849 cuando la Inspección Educativa nace en España como institución diferenciada, estableciéndose en esta norma sus funciones, las cuales tienen como intención permitir a la Administración satisfacer su necesidad de conocer lo que ocurre en el ámbito educativo, para lo cual se crea un cuerpo integrado por personas con la debida cualificación y experiencia para ello.

Así, se establece un rol de la Inspección centrado fundamentalmente en proporcionar información en sentido ascendente, con el fin de proporcionar datos y evidencias a la Administración que permita la toma de decisiones y que al mismo tiempo garantice el cumplimiento de la norma.

El Reglamento de 20 de mayo de 1849, con el que se desarrolla lo establecido en el Real Decreto antes citado, establece entre las tareas inspectoras algunas que son de interés dentro del tema que nos ocupa, como son las de examinar los métodos utilizados por el maestro y sugerir mejoras en los métodos de enseñanza.

Sin embargo, y a pesar de dichas intenciones, la realidad es que la labor de la Inspección en esta época pone su acento en la función administrativa, de vigilancia y control, sin poder apreciarse el carácter pedagógico de la misma.

De este modo, durante la segunda mitad del siglo XIX y comienzos del XX se aprecia una leve acción orientadora y pedagógica de la inspección en los centros, tarea que se ve sobrepasada por funciones meramente administrativas ante las autoridades educativas. 
Sin embargo, y a pesar de esto, son varios los inspectores que en estas décadas realizan diversas contribuciones al ámbito de la pedagogía, aún en los inicios de esta disciplina, escribiendo los primeros textos y tratados pedagógicos que sirvieron de consulta y apoyo para una gran cantidad de docentes durante varios decenios.

No será hasta la segunda década del siglo XX cuando comience a replantearse el papel de la Inspección de Educación, coincidiendo con la incorporación de nuevas ideas sobre la función de ayuda, orientación y apoyo que ésta debía desarrollar en su labor con los docentes. En consecuencia, desde los sectores más modernizadores se apuesta por un viraje hacia un mayor acompañamiento pedagógico, lo que a la larga supone un cambio en las tareas a desempeñar, así como el peso de las mismas. Así, como señala Hernández (2019):

La Inspección era considerada por este movimiento pedagógico renovador como una pieza clave del engranaje de cambio de la escuela española, si bien encontraba severas dificultades para sortear y superar un modelo de inspector tradicional, jerárquico, funcionarial, más orientado a la garantía del estricto y severo cumplimiento de las leyes que a la tarea de ayuda y orientación a los muchos maestros que carecían de preparación, motivación y condición social y salarial digna (p. 8).

Hasta el año 1936 encontramos novedades en cuanto a la formación, especialmente pedagógica, de los inspectores en nuestro país, todo ello apoyado en el desarrollo de una nueva cultura escolar impulsada tanto desde la Administración como desde el aula y en consonancia con lo que ocurría en diversos países europeos y americanos, llegando a su auge durante la II República. Ejemplo de ello es la creación de los Centros de Colaboración Pedagógica, cuya función era el fomento del intercambio de experiencias entre docentes de una comarca con la ayuda del inspector, o la publicación de los Boletines de Educación a nivel provincial por parte de los inspectores, con la misión de trasmitir experiencias innovadoras y crear debate sobre diversos aspectos de la escuela primaria y sus maestros. Se concibe de este modo una inspección más técnica, más centrada en la orientación que en el control, y todo ello sustentado sobre una formación sólida.

A partir de 1936 se produce un cambio en el paradigma de la labor inspectora, de manera que a lo largo de la dictadura franquista se promueve una inspección centrada en el control y la vigilancia, orientada al estricto cumplimiento de la normativa educativa y la ideología imperante, y desapareciendo, de forma práctica, su autonomía para acompañar los procesos de mejora e innovación escolar.

No será hasta 1970 cuando se materialicen los distintos movimientos de renovación pedagógica que venían produciéndose desde los años 50 del siglo pasado, coincidiendo con un cierto aperturismo del régimen en el ámbito educativo. Así, la Ley General de Educación de 1970 dedica parte de su articulado a la Inspección, señalando entre sus funciones la de "Asesorar a los profesores de los centros estatales y no estatales sobre los métodos más idóneos para la eficacia de la enseñanza que imparten”, y la de “Colaborar con los Institutos de Ciencias de la Educación en la organización de cursos y actividades para el perfeccionamiento y actividad del personal docente”. Estos cambios, como señala Hernández 
(2019) no se llevaron a cabo de manera sencilla, ya que a este planteamiento se oponen tanto diversos sectores del régimen como los sectores más democráticos y aperturistas.

En cualquier caso, ese paso adelante de la Ley General de Educación de 1970 supuso un intento de cambiar el papel de la Inspección en los centros educativos, especialmente en lo relativo a la formación del profesorado, si bien no llegó a desarrollarse totalmente en la práctica.

En el final del siglo XX, las distintas leyes educativas promulgadas a partir de la Constitución de 1978 han ido estableciendo, y ampliando, las funciones de la Inspección Educativa en el sistema educativo, de manera que en todas ellas podemos encontrar similitudes a la hora de enunciarlas. Sin embargo, se produce un hecho clave que modifica el papel que ésta asume en el ámbito de la innovación, como es su sustitución por los centros de profesores y los equipos de orientación educativa en las labores de asesoría pedagógica y didáctica.

En definitiva, el devenir histórico de la Inspección Educativa nos pone de manifiesto el papel que ésta ha jugado en el ámbito de la innovación, poniéndose de manifiesto cómo, salvo en periodos muy concretos de la historia de nuestro país, su tarea fundamental ha estado centrada en la supervisión y el control más que en el asesoramiento técnico y pedagógico a los centros y docentes.

\section{El panorama actual en España}

Uno de los hechos diferenciales de nuestro país es la descentralización administrativa a nivel general, y dentro de ella la educativa a nivel particular, lo que provoca que dentro del marco de las leyes de ámbito estatal, las distintas administraciones son las que establecen la organización y funcionamiento de sus servicios de Inspección de Educación.

De este modo, aunque todas tienen una base común, podemos encontrar elementos diferenciadores que determinan el modo en el que se lleva a cabo las tareas de supervisión, evaluación y asesoramiento, así como el énfasis que se pone en cada una de ellas.

Es en los distintos planes de actuación de las inspecciones educativas de cada comunidad autónoma donde podemos encontrar qué papel y qué tareas asumen éstas en el ámbito de la innovación educativa.

En el caso de Andalucía, la Orden de 19 de julio de 2019, por la que se establece el Plan General de Actuación de la Inspección Educativa de Andalucía para el período 2019-2023 señala en su preámbulo que:

La Inspección Educativa de Andalucía se enfrenta a nuevos retos que requieren de un proceso colectivo de reflexión que permita por un lado, consolidar el modelo de intervención adoptado en los Planes de Actuación anteriores; y por otro continuar con la supervisión y evaluación de centros y servicios educativos, fomentando que sus órganos, en el uso de su autonomía, formulen propuestas generadoras de los cambios y transformaciones necesarias para contribuir al éxito 
educativo del alumnado, a la reducción del abandono escolar y al aumento de titulados en la educación obligatoria y postobligatoria.

Para ello, este Plan se plantea como uno de sus objetivos el "Detectar e impulsar la divulgación de las buenas prácticas desarrolladas y acreditadas en los centros docentes, servicios, programas y actividades del sistema educativo, así como de las innovaciones educativas" para lo cual se fija como línea estratégica la "Evaluación de centros docentes, servicios, programas y actividades del sistema educativo de la función directiva y de la práctica docente a través del análisis de la organización, el funcionamiento y los procesos de enseñanza y aprendizaje para la mejora educativa, con efecto de reconocimiento de logros, introducción de innovaciones y, en su caso, de propuesta de mejora.”.

Este objetivo que citamos es concretado en la Resolución de 24 de julio de 2019, de la Viceconsejería, por la que se aprueban las Instrucciones para el desarrollo, la dirección y la coordinación del Plan General de Actuación de la Inspección Educativa y el Plan de Formación para el Perfeccionamiento y Actualización Profesional, para el curso escolar 2019-2020 de la siguiente manera:

1. Detectar y analizar buenas prácticas que se desarrollan en los centros y servicios educativos para su conocimiento, divulgación y transferencia.

2. Promover el conocimiento de buenas prácticas educativas por parte de la Administración educativa y la sociedad en general.

3. Detectar prácticas relevantes que se desarrollan en los centros y servicios educativos para su conocimiento, divulgación y generalización.

Con el fin de alcanzar los objetivos planteados se establece una actuación prioritaria centrada en la supervisión y evaluación de centros y servicios educativos que se focalizará sobre los procesos de aprendizaje y evaluación del alumnado, las líneas metodológicas establecidas y, una vez finalizada, en las necesidades detectadas en los centros y servicios con especial atención a las del alumnado, las cuales se vincularán con la formación del profesorado como vía de respuesta a las mismas, y estableciendo la visita al aula como instrumento clave para el desarrollo de la misma y conocimiento de lo que ocurre en los centros.

Esta actuación finaliza con la presentación de un informe del Equipo de Inspección de Zona, a la vista del cual y de las propuestas de mejora incluidas en el mismos, los centros docentes de la muestra deben elaborar un plan de trabajo que incluirá actuaciones para dar cumplimiento a las mismas y, en su caso, introducir innovaciones; este plan se presenta al Equipo de Inspección de Zona correspondiente, siendo sus líneas de trabajo objeto de seguimiento y asesoramiento por, al menos, la inspección de referencia a lo largo del siguiente curso escolar.

El País Vasco por su parte recoge en su Plan Trienal 2017-2020 que la labor de la Inspección Educativa ha ido avanzando hacia un nuevo modelo que viene a constituir una exigencia de mayor dedicación a las labores de asesoramiento, orientación e información.

En el proceso de reflexión estratégica para la elaboración de este Plan se señala como una de las oportunidades detectadas los propios cambios que se están produciendo en el sistema 
educativo, tales como la implantación del nuevo currículo, que permite la intervención de la Inspección como un agente motor de la innovación.

Así, se pretende que la intervención contribuya eficazmente a la garantía del derecho a la educación y a promover que los centros educativos den una respuesta de calidad que lleve al éxito de cada uno de sus alumnos y alumnas en los procesos de aprendizaje, para lo cual esta intervención no debe limitarse al mero control normativo, sino que debe incluir el acompañamiento y apoyo a los centros en el desarrollo de sus proyectos educativos, promoviendo procesos de reflexión y autoevaluación, participando en los procesos de evaluación externa y emitiendo propuestas que contribuyan a la mejora de su funcionamiento y de su respuesta educativa.

En relación con la innovación educativa, se fija en este Plan trienal como línea básica de trabajo la contribución a la implantación de los cambios metodológicos en los centros, encargándose la Inspección de Educación de acompañar a los centros en esta travesía, en coordinación con los Berritzegunes (servicios de apoyo, instrumentos educativos para la innovación y mejora de la educación), realizando un seguimiento de la implantación de las nuevas propuestas metodológicas en diferentes muestras de centros y realizando las acciones que se deriven de la información obtenida mediante dicho seguimiento.

En el caso de Extremadura, se fija como uno de los objetivos de la Inspección Educativa el de "articular un sistema de asesoría y colaboración con los centros que facilite el tratamiento y desarrollo de las competencias clave, la aplicación de las TIC y la progresiva implantación del plurilingüismo y de los programas educativos conducentes al éxito escolar”.

Para ello, y de acuerdo con la Ley Orgánica 2/2006, 3 de mayo, de Educación, la Ley 4/2011, de 7 de marzo, de Educación de Extremadura, se fija como una de las funciones de la Inspección de Educación el "fomentar y divulgar la experimentación, la innovación y la investigación educativas”.

Así, como concreción de lo anterior, el Plan Director de Actuación de la Inspección en Extremadura para el trienio 2017-2020 establece como uno de sus objetivos el de "contribuir a la mejora de la calidad de las enseñanzas, a través de la supervisión, control, evaluación, asesoramiento e información, en centros y servicios educativos, sobre factores que inciden en los procesos de enseñanza - aprendizaje y en los resultados de los centros”.

Para la consecución de este objetivo se plantea como actuación la supervisión, control, evaluación y asesoramiento en el ámbito de la organización y funcionamiento de los centros educativos, atendiendo entre otros aspectos a la mejora de los procesos de enseñanza, de los resultados de aprendizaje y en general al éxito escolar del alumnado, y centrándose en el desarrollo de los planes, programas y proyectos que se implanten en el centro y en el adecuado uso de los recursos para lograr el fin de los mismos, con especial incidencia en aquellos proyectos que contribuyen al éxito escolar. 
Para terminar, citaremos el caso de la comunidad autónoma de Cataluña, la cual desde su propia Ley 12/2009, de 10 de julio, de Educación establece el papel que asume la Inspección Educativa en el ámbito de la innovación, y partiendo de su pretensión de fijar un marco que "potencia la innovación pedagógica sistemática y estructurada, el reconocimiento de las buenas prácticas educativas con el fomento y el apoyo del liderazgo educativo, la formación del profesorado, las infraestructuras digitales del centro y la previsión de centros de referencia pedagógica.” (Preámbulo)

Para ello, entre otros aspectos a lo largo de la Ley, incluye entre las funciones de la Inspección de Educación la de "participar en el desarrollo de acciones para la mejora de la práctica educativa y del funcionamiento de los centros, así como de los procesos de reforma e innovación educativa.” (art. 178)

Se puede apreciar como en el caso catalán puede observarse un cambio en el uso de los términos, no se utiliza "asesorar" sino "participar”, lo que de facto implica la necesidad de colaboración y coordinación con los centros y otros servicios educativos, y no únicamente una visión de carácter externo.

Sin embargo, la Orden ENS 303/2015 de 21 de septiembre, sobre el reconocimiento de la innovación pedagógica establece en su artículo 5 que corresponde a la Inspección de Educación el supervisar y evaluar la aplicación de las modalidades de innovación pedagógica, y determinar la contribución de estas modalidades en la mejora de los resultados de los centros, no señalándose nada en relación con la participación de ésta en dichos procesos de innovación, asumiendo tareas de supervisión y evaluación.

Vistos los ejemplos anteriores, podemos concluir que la Inspección de Educación en nuestro país cuenta con un marco normativo que le permite participar en los procesos de innovación educativa que se desarrollen en los centros, si bien los propios planes de actuación de inspección no concretan el modo en que se debe desarrollar dicha participación, aunque se planifica el desarrollo de actuaciones de supervisión y evaluación como base necesaria para el asesoramiento a los centros en el desarrollo de sus procesos de enseñanza - aprendizaje.

Para finalizar este apartado, y tras revisar algunos planes de actuación de nuestro país, podemos destacar algunos elementos comunes:

- Se coincide en la necesidad de implementar cambios en el modelo de inspección, con un mayor peso de la evaluación y el asesoramiento, pero sin dejar de lado la supervisión, con el fin de actuar como agentes de los procesos de innovación.

- Junto con la evaluación de manera externa, se aboga por potenciar la autoevaluación de centros.

- Se insiste en la necesidad de promocionar la implantación de cambios metodológicos en los centros y aulas, así como de los procesos de evaluación y asesoramiento de los mismos.

- Se promueve la detección y divulgación de buenas prácticas desarrolladas en los centros y aulas. 


\section{Tendencias en el contexto europeo}

En el ámbito europeo viene mostrándose en los últimos años un interés creciente por la innovación educativa y por el desarrollo de políticas dirigidas a su desarrollo.

Uno de estos estudios, "Study on supporting school innovation across Europe" se encarga de ofrecer una revisión exhaustiva de las políticas de investigación e innovación escolar existentes en los 28 Estados miembros de la UE, incluyendo diversos estudios de casos de escuelas exitosas ubicadas en regiones desfavorecidas de la UE.

Este estudio fija las características clave con las que cuentan las escuelas innovadoras (liderazgo escolar de apoyo y distribuido; preparación, compromiso y profesionalidad docentes; una visión compartida para el desarrollo escolar junto con estrategias internas y planes de acción; implicación de los estudiantes como participantes activos en el proceso de cambio; apertura hacia la comunidad), así como los requisitos que debe cumplir un sistema educativo que pretende innovar (un marco de políticas coherente que cubra áreas clave respaldado por una visión a largo plazo para la incorporación de la innovación y garantizar la transformación de todo el sistema; políticas co-construidas por parte interesadas clave; coherencia y claridad en las iniciativas y políticas y sus relación; estrategias políticas respaldadas por iniciativas de apoyo y medidas concretas; capacidad de las escuelas para gestionar y mantener el cambio).

Para ello, las evidencias recogidas en este estudio demuestran que las escuelas tienen más probabilidades de desarrollar una cultura de innovación cuando:

- Poseen suficiente autonomía sobre el plan de estudios, los métodos de enseñanza, la gestión del personal y los recursos financieros.

- Son horizontalmente responsables.

- Tienen la capacidad y las oportunidades para participar en asociaciones y redes.

- Están respaldados por sistemas de desarrollo profesional relevantes para maestros y líderes escolares.

De este modo, se concluye que los países que logran transformar sus sistemas educativos son aquellos en los que se garantiza una inversión sostenible en las áreas clave cubiertas por las reformas educativas. Estos países también fortalecen su marco de evaluación, promoviendo una cultura de autoevaluación y desarrollando la capacidad de las partes interesadas en educación para usar e interpretar los datos que se obtienen.

En este panorama, se señala como un elemento clave el compromiso de la Inspección en el diálogo y los procesos de aprendizaje con las escuelas, apoyándolas en el proceso de innovación. Al mismo tiempo, se considera la evaluación externa, en la que la Inspección participaría como uno de sus agentes, como uno de los componentes clave de una estrategia de garantía de calidad. 
De esta forma, las inspecciones enfocadas a promover la mejora se definen con base en las siguientes características:

- Todavía cumplen un rol de salvaguarda público, moderando la calidad de la autoevaluación escolar, pero pueden hacerlo sobre una base más flexible en lugar de un ciclo fijo, lo que les permite intervenir rápidamente cuando se aprecian señales que indican que una escuela debe ser inspeccionada con urgencia.

- Pueden asumir una menor estandarización y mayor flexibilidad en su tarea, personalizando la manera en que inspeccionan e informan sobre y a las escuelas, $\mathrm{y}$ haciéndolo de una forma más completa, narrativa e individualizada.

- Pueden desarrollar un estilo de compromiso que sea más parecido a la formación que a la rendición de cuentas, con niveles más altos de diálogo profesional e integrado en el proceso de ayuda a las escuelas, las cuales pueden aprender de la experiencia con la que cuentan los inspectores de su práctica en otros centros e instancias.

Junto con lo anterior, con el fin de dar respuesta a las inquietudes derivadas de la necesidad por determinar el papel y el impacto de la Inspección en la mejora de las escuelas y redes escolares, son diversos los estudios que se han venido realizando en los últimos años, tales como el proyecto europeo "School Inspections", así como las asambleas generales y talleres que periódicamente desarrolla la Standing Internacional Conference of Inspectorates (SICI), lo que pone de manifiesto, como ya se ha señalado, el interés creciente por la innovación educativa desde el ámbito de la Inspección, pudiendo destacar varias ideas recurrentes en los trabajos y estudios realizados:

- Los factores que conducen al éxito en la enseñanza, a mejorar el ambiente de aprendizaje así como los resultados son aquellos que la Inspección contempla en sus actuaciones.

- El asesoramiento y la orientación en la aplicación de las normas educativas con el fin de mejorar la calidad de la enseñanza es uno de los ejes fundamentales de los nuevos modelos de Inspección.

- Los consejos y orientaciones que proporciona la Inspección en sus actuaciones son un buen punto de partida para el desarrollo y mejora de las escuelas.

- Importancia de la Inspección como agente de evaluación externo al centro en coordinación con una autoevaluación realizada por parte de los centros.

- Importancia de la creación de redes de organizaciones e instituciones educativas coordinadas lideradas, guiadas y asesoradas por la Inspección.

- Desarrollo de funciones que potencien la autonomía de los centros, propicien una reflexión sobre nuevas formas de enseñar y aprender, y ayude a los docentes a buscar estrategias para responder a las necesidades futuras del alumnado.

\section{Una propuesta de trabajo}

La cuestión clave que esta institución debe plantearse es precisamente qué tipo de inspección se pretende, tal como planteamos en el XIV Congreso Nacional de Inspectores de Educación 
“Innovar en educación - Inspección Educativa, celebrado en 2016 (Valladolid, 19 al 21 de octubre).

Una vez que sus funciones se fijan a través de una Ley Orgánica y éstas se desarrollan a través de los diferentes planes de actuación que establecen las Administraciones educativas, es necesario que estos planes fijen claramente las actuaciones a desarrollar por la Inspección Educativa, ya que son éstas las que determinan el modelo de Inspección que se pretende, bien un modelo de Inspección orientado a que los centros introduzcan cambios organizativos, funcionales y/o metodológicos, o un modelo en el que la Inspección se ocupa única y exclusivamente de velar por el cumplimiento de la norma.

En este sentido debemos tener en cuenta el cambio paradigmático que se está produciendo en el ámbito educativo general, lo que sin duda obliga a que la Inspección deba replantearse sus funciones y el rol que debe asumir.

Así, la Inspección no puede ni debe única y exclusivamente llevar a cabo funciones de supervisión y control, aun siendo esta una de sus funciones esenciales, ya que quedarse ahí podría provocar el dejar de lado otro muchos aspectos de gran importancia; la Inspección no sólo debe velar porque las decisiones que tomen los centros se ajusten a la norma, sino que también debe valorar el porqué de esas decisiones y los efectos que éstas tienen sobre los diversos procesos que llevan a cabo los centros educativos.

En este sentido, como señala Vázquez (1993), aquellos sistemas donde existe una clara relación entre supervisión y asesoramiento son sistemas más evolucionados. De este forma, no podemos pretender que se gire visita a un centro, se le señale los incumplimientos normativos y se vuelva una vez pasado un tiempo para ver si se ha ajustado a la norma. Cuando un centro no cumple con lo prescriptivo puede deberse a dos razones, por desconocimiento o por incapacidad para cumplirla; esto lleva a la Inspección a la necesidad de desarrollar funciones de asesoramiento técnico, que permita dar a conocer a los centros la razón por la que no se ajustan a la norma y a partir de ahí ayudarles a que puedan hacerlo de la mejor manera posible.

Al mismo tiempo, la Inspección de Educación no puede plantearse que con solo "estar” se van a producir cambios en los centros educativos, sino que se precisa una adecuada evaluación y un asesoramiento y seguimiento posterior ajustado a las conclusiones de la misma.

Es importante, antes de establecer una propuesta sobre el papel que debe asumir la Inspección en relación con la innovación educativa, delimitar el concepto de la misma, ya que dicha concepción va a determinar el modo en que ésta actúe.

El término innovación es un término polisémico, abarcando desde el concepto de reforma educativa en sentido amplio, hasta el desarrollo de mejoras sobre lo que se lleva a cabo, es decir, innovar no representa solo a algo nuevo sino también a la actualización o progreso de lo que se viene haciendo con el objetivo de mejorar los resultados que se obtienen. 
Partiendo de esta conceptualización del término innovación, establecemos tres niveles en los que la Inspección puede colaborar para el desarrollo de los procesos de innovación educativa:

- A nivel macro, participando en la elaboración, desarrollo y evaluación de los programas de innovación que ponen en marcha las Administraciones educativas;

- a nivel meso, mediante la evaluación y asesoramiento en los proyectos de innovación pedagógica que ponen en marcha los centros educativos;

- y, por último, a nivel micro, donde se identifican y evalúan practicas innovadoras a nivel de aula con el objetivo de asesorar y promover su difusión.

En el caso del nivel macro, la posición de la Inspección de Educación en el sistema educativo y su conocimiento de una gran cantidad y variedad de centros, hace que esta institución se convierta en un medio para informar a las Administraciones educativas de lo que ocurre en ellos, con el fin de facilitar la toma de decisiones y promover la introducción de determinadas innovaciones a través de planes y programas institucionales.

El nivel meso es donde, quizás, la Inspección Educativa puede tener un mayor impacto en el desarrollo de procesos de innovación educativa, jugando un papel clave en la coordinación de centros y servicios educativos. Así, a partir de la supervisión y evaluación de los centros y la detección de sus necesidades, debe ser posible establecer planes conjuntos que permitan, tanto divulgar buenas prácticas de cambio e innovación que se estén llevando a cabo, como para fijar planes de formación.

Este papel como coordinadores de los servicios externos es, por tanto, clave, como conocedores de la realidad de los centros y su capacidad para determinar las fortalezas y oportunidades de mejora de estos.

Hoy más que nunca se puede apreciar como el cambio metodológico es necesario, pero este cambio no puede producirse exclusivamente con la publicación de leyes y normas, de arriba a abajo, sino que debe desarrollarse desde los centros y las aulas, de abajo a arriba, con el objetivo de lograr mejores procesos de enseñanza y por tanto una mejora de los aprendizajes del alumnado.

De esta manera, el papel de la Inspección debe ser la de participar, asesorar y apoyar los cambios metodológicos que se llevan a cabo en los centros, favoreciendo la innovación a través de la autonomía pedagógica y organizativa con la que cuentan los centros, sin que ello suponga dejar de velar por los derechos de todos los sectores de la comunidad educativa.

Junto a lo anterior, se hace necesario incidir y promover el análisis y la reflexión de los centros sobre los procesos que desarrollan y los resultados que obtienen, es decir, procesos de autoevaluación, con el fin de que estos puedan introducir cambios y adoptar decisiones orientadas a la mejora. 
No podemos olvidar el papel fundamental que juega en todo esto la Dirección de los centros, elemento también recogido como factor de calidad en la LOE, ya que es con quien, generalmente, se establece una relación más directa por parte de la Inspección, mientras que con el resto de miembros del centro se desarrolla una relación mediada. Así, en la medida en que exista una mayor coordinación, intercambio y entendimiento entre estos dos agentes, será posible una mayor capacidad de generar cambios en los centros por parte de la Inspección.

En esta línea, podemos citar la experiencia desarrollada por parte del Equipo de Inspección de Zona de Cádiz I, que abarca las localidades de Cádiz, Puerto Real y El Puerto de Santa María, durante los cursos 2015 a 2019, la cual involucró a 5 inspectores e inspectoras, 3 Equipos de Orientación Educativa, varios asesores y asesoras del Centro de Profesorado de Cádiz y 35 direcciones de centros públicos de educación infantil y primaria. En ella, tras la detección de necesidades de formación en los centros durante el desarrollo del nuevo currículo de la Educación Primaria, la Inspección Educativa se encargó de coordinar, a través de formación en centros y un grupo de trabajo, la labor de desarrollar los procesos de evaluación del alumnado, y cuyos resultados han permitido introducir innovaciones y cambios metodológicos a nivel de centro y localidad, de manera que los procesos de investigación educativa pueden llegar a provocar cambios a nivel macro en un futuro próximo.

Por último en este nivel, la Inspección debe jugar el papel de difundir las buenas prácticas que se hayan detectado en los centros, favoreciendo el intercambio de experiencias y el conocimiento de las mismas por parte de la Administración y otros centros, de manera que éstas puedan generalizarse, tras demostrar su eficacia, una vez adaptadas a las características y contextos particulares.

Finalmente, a nivel micro la Inspección debe desarrollar dos funciones clave, por un lado evaluando y asesorando aquellos procesos de innovación que se lleven a cabo a nivel de aula, y por otro lado, promoviendo la generalización a nivel de centro de aquellas buenas prácticas que se detecten a nivel de aula. En este sentido, la intención no es otra que la de acabar con un problema habitual como es la puesta en marcha de prácticas innovadoras por parte los docentes nivel individual, sin coordinación con otros miembros del claustro ni darse a conocer en el mismo centro, situaciones que deben evitarse si se pretenden cambios metodológicos que realmente tengan impacto sobre el alumnado.

En cualquier caso, y para terminar, no podemos obviar la necesidad de mejorar la formación pedagógica y didáctica de los miembros de los servicios de Inspección, con el fin de poder desarrollar adecuadamente sus funciones de evaluación y asesoramiento, permitiéndoles conocer nuevos modelos y propuestas metodológicas, así como experiencias y proyectos innovadores a nivel local, autonómico, nacional e internacional, tanto para contar con una adecuada base en los procesos de valoración como para difundir éstas en los centros en los que se actúa. 


\section{Referencias}

Buj Romero, A. (1992), Inspección técnica, sistema educativo e innovación en Teoría de la Educación, Vol. IV, nº 4, pp. 185-207.

Cáceres Reche, M.P., Hinojo Lucena, F.J. y Aznar Díaz, I. (2007). Evolución histórica de la Inspección Educativa a través de los principales referentes legales. Avances en Supervisión Educativa, 6.

Dumcius, R., Whittle, M., Huttova, J., Sirova, H., Sternadel, D., Mackonyte, G., Jonaviciene, D., Junas, P., y Buinauskas, D. (2018). Study on Supporting School Innovation Across Europe. Comisión Europea.

Escudero, J.M. y Moreno M.A. (2012). Mejorar la educación, la autonomía de los centros y el servicio de Inspección Educativa. Avances en Supervisión Educativa, 17.

Esteban Frades, S. (2007). Reflexiones sobre las antinomias de la Inspección Educativa en España. Un problema sin resolver. Avances en Supervisión Educativa, 6.

Esteban Frades, S., Sarasúa Ortega, A., Senrentill Rubio, J. y Zulueta Castañeda, M. (2016). Mesa redonda; papel de la Inspección en la innovación educativa. Avances en Supervisión Educativa, 26.

Estefanía Lera, J.L. (2017). La Inspección ante la innovación educativa. Avances en Supervisión Educativa, 27.

Hernández Díaz, J. (2019). La Inspección Educativa y la cultura escolar en España. Génesis, proceso constituyente y actualización de funciones. Aula, 25, 59-89.

Martínez Bonafé, J. (2008). Pero ¿qué es la innovación educativa? Cuadernos de pedagogía, $375,78-82$.

Sarasúa Ortega, A. (2019). La Inspección de educación, un futuro incierto. Aula, 25, 91-104. doi: 10.14201/aula20192591104

School Inspections (2011-2013). Studying the impact of school inspections on improvement of schools. Recuperado de https://tinyurl.com/vepgk75

Secadura Navarro, T. (2011). El referente de la Inspección Educativa: el centro docente versus el sistema educativo. Avances en Supervisión Educativa, 15.

Vázquez Gómez, G. (1993). Supervisión y asesoramiento. En E. Soler Fierrez, Fundamentos de supervisión educativa. Madrid: La muralla. 
Referencias Normativas

Ley Orgánica 2/2006, de 3 de mayo, de Educación (Documento consolidado). BOE de 4 de mayo de 2006.

Ley 12/2009, de 10 de julio de Educación. DOGC de 16 de julio de 2009.

Orden ENS/303/2015, de 21 de septiembre, sobre el reconocimiento de la innovación pedagógica. DOGC 30 de septiembre de 2015.

Orden de 19 de julio de 2019, por la que se establece el Plan General de Actuación de la Inspección Educativa de Andalucía para el período 2019-2023. BOJA de 30 de julio de 2019.

Resolución de 24 de julio de 2019, de la Viceconsejería, por la que se aprueban las Instrucciones para el desarrollo, la dirección y la coordinación del Plan General de Actuación de la Inspección Educativa y el Plan de Formación para el Perfeccionamiento y Actualización Profesional, para el curso escolar 2019-2020. BOJA de 1 de agosto de 2019.

Resolución de la Viceconsejera de Educación por la que se aprueba el plan general trienal de la Inspección de Educación para los cursos 2017-2018, 2018-2019 y 2019-2020. Recuperado de https://tinyurl.com/u3trtwx.

Instrucción de la Secretaría General de Educación por la que se establece la organización y funcionamiento y se aprueba el Plan Director de Actuación de la inspección de educación de Extremadura para el trienio 2017-2020. Recuperado de https://tinyurl.com/tmdlwje. 